\title{
Specular Flow and Shape in One Shot
}

\author{
Yair Adato \\ http://www.cs.bgu.ac.il/ adato
}

Ohad Ben-Shahar

http://www.cs.bgu.ac.il/ ben-shahar

\author{
Department of Computer Science, \\ Ben-Gurion University of the Negev, \\ Israel
}

\begin{abstract}
The reconstruction of specular shape from images is a very challenging task, especially when the illumination environment is unknown. In such cases, the problem becomes more tractable once the camera observes relative motion between the object and the environment, which induces a type of optical flow field that has become known as specular flow. Unfortunately, however, the estimation of specular flow from image sequences is even more challenging, putting in question the effectiveness of the shape from specular flow approach as a whole. Here we show that instead of the traditional (and somewhat futile) process of first estimating the specular flow and then using it for the recovery of specular shape, an approach that addresses these two inference problems simultaneously improves the estimation of both structures. We formulate the problem in a variational setting, we identify and address numerical issues unique to its application on specular flows, and we employ a polar representation of motion, all to result in the first ever practical method to compute specular shape from real image sequences under unknown illumination.
\end{abstract}

\section{Introduction}

An image of a purely specular object is just a distortion of its surrounding illumination environment, hence one can create any given image from any given specular object by a proper manipulation of the environment [ $⿴ 囗 ⿱ 一 一])$. Therefore, when only little (or nothing) about the illumination environment is known, inferring the geometrical structure of a specular surface becomes a hard problem. While most existing algorithms for specular shape reconstruction indeed assume known or calibrated illumination, recent studies have addressed the problem without such knowledge by exploiting relative motion between the observed object, the camera, or the environment. In the image plane such motion induces a specular flow - the optical flow of observed specular reflections - which has been shown to be independent of environment content and therefore to facilitate shape reconstruction (e.g., [प, $\square, \boldsymbol{Q}, \square, \square]$ ).

The shape from specular flow (SFSF) approach follows the intuitive hierarchical thinking that was advocated by Marr $[\square]$ and guided generations of researchers of structure from motion (and others): First, estimate the relevant (specular) flow in the image plane. Next, exploit it for (specular) shape recovery. Theoretically, this framework allows dense reconstruction of any general smooth specular object assuming no prior knowledge about the object or the content of the illumination environment.

Unfortunately, there are severe practical difficulties to this type of approach. The most critical of which is the ability (of the lack thereof) to reconstruct specular flow reliably from 
image sequences. As argued in the past, specular flows are optical flows by definition and yet, they possess certain properties that prevent optical flow algorithms from estimating them reliably or reasonably [ $[$ ] . It is well known, for example, that specular flows undergo unique singularities at (or near) the projection of parabolic lines, where reflected features are created and annihilated in pairs $[\square, \square, \square]$. In practice, this so called parabolic singularity [ $[$ ] drives virtually all optical flow algorithms into global qualitative errors across the image plane. The same problem is revealed in related methods also, and is coped with by assuming no

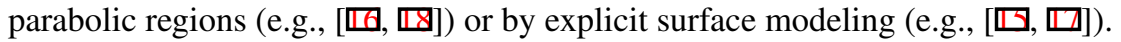

It should be noted that even if the flow is assumed to be known and perfect (that is, without errors), the reconstruction process itself is not devoid of practical issues, since its reliance on the solution of partial differential equations requires enough initial conditions. How to extract these data from the specular flow (or from other image cues) remains an open question.

In this work we propose an alternative approach that overcomes many of the problems above. The key element in our proposal is the estimation of specular flow and surface geometry simultaneously. The rational is simple - each structure incorporates constraints that could assist in the estimation of its counterpart, thereby limiting the potential deviation of both from the desired outcome. We show that this strategy becomes possible by endowing the classical optical flow assumptions for optical flow estimation (e.g., brightness constancy, piecewise smoothness) with additional constraints that emerge from surface geometry considerations (e.g., object smoothness and expected behavior at surface boundaries) and those that reflect the imaging model that links the shape to the specular flow. As it turns out, formulating the problem in this way allows us to suggest an algorithm which does not require any prior initial (shape) conditions. To our best knowledge, the suggested algorithm is the first to recover general dense specular shape (with no self-reflections), including objects that contain parabolic lines, directly from image sequences under unknown illumination. We do note that since the relationship between the flow and the shape suffers an intrinsic ambiguity, the recovery is only up to a family of surfaces.

\section{Previous work}

Most of the specular shape from motion studies have considered sparse illumination environments, and quite often just a single light source. When motion is incorporated (e.g., by changing the viewpoint or moving the object or the light source) under calibrated illumination, one can track the isolated specular highlights to exploit constrains between the surface normal, viewing direction, and the illumination direction $[\square, \square, \square]$. This facilitates shape reconstruction in sparse locations only, a limitation which was initially addressed by extended motion sequences, object modeling, or deep regularizations (e.g., [凹, 四, 四]).

To cope with dense reconstruction of specular shape in unknown natural illumination environment, a recent line of research has proposed to exploit the specular flow $[\square, \square, \square]$. Perhaps the most rigorous of these recent studies is the Shape-from-Specular-Flow (SFSF) approach $[\square, \square, \square]$, where the authors have derived a system of non-linear PDEs (SFSF equation) which expresses the relationship between the specular flow, the shape of the specular object, and its motion relative to the environment. Solving SFSF equation provides the sought-after shape assuming a particular imaging model and enough initial conditions. In most cases, however, it is not known how to solve SFSF equation or how to obtain the initial conditions. Some progress related to the former problem was presented by Canas et al. [ $\mathrm{\theta}]$, who proposed to represent the unknown shape via the field of its reflection vectors, $\mathbf{r} \in S^{2}$ 


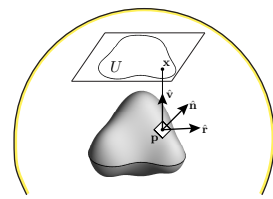

(a)

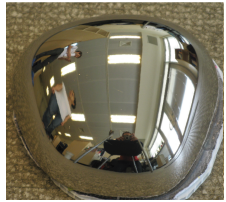

(b)

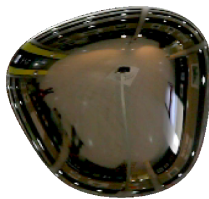

(c)

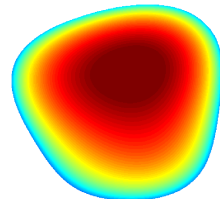

(d)

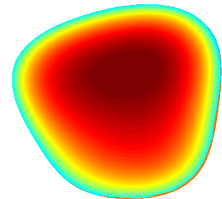

(e)

Figure 1: Evaluation of the basic algorithm. (a) The setup: a specular surface is observed orthographically in a distant unknown illumination environment. (b) A real specular object with ground truth data from [0]. (c) A sample frame observed from the object. (d) Ground truth shape data. (e) Estimate shape using our basic algorithm.

(see Fig. 1-a). Interestingly, although this field is a straight forward extension of the surface normal field (when the viewing direction is fixed), this apparently simple change of representation yields a linear form for the SFSF equation:

$$
(D \mathbf{r}(\mathbf{x})) \mathbf{u}(\mathbf{x})=\omega \times \mathbf{r}(\mathbf{x}),
$$

where $\operatorname{Dr}(\mathbf{x}) \triangleq \partial \mathbf{r}(\mathbf{x}) / \partial \mathbf{x}$ is Jacobian of the reflection map, $\mathbf{u}=(u, v)$ is the specular flow, $\omega$ is the environment rotation axis. Being linear, this version of the SFSF equation offers better opportunities for easier solutions in a wider range of cases, although initial conditions are still needed for each integral curve of the specular flow.

Regardless of which SFSF one attempts to solve, one question remains - how can one measure or estimate the specular flow reliably and accurately enough from image sequences. Since specular flows are, by definition, optical flows [ $\square]$, it was first proposed to employ optical flow algorithms for this task (e.g., [四, 四). Unfortunately, however, this is a poor choice. Indeed, as was argued recently [G], existing optical flow algorithms fail on specular flow data primarily because their regularization terms misrepresents the parabolic singularities and the significant variations in the flow magnitude. Alternatively, it was also suggested to avoid estimating the dense specular flow directly and instead to find a sparse set of corresponding reflection points in the image sequence which are far enough from parabolic singularities $[\square]$. Here, however, not only that finding correspondence between specular reflections is difficult, the completion of data between these points requires surface modeling (e.g., as quadratic forms), which inevitably introduce additional inaccuracies.

As implied above, in this work we suggest to couple the estimation of dense specular flow and the reconstruction of the corresponding specular shape as tightly as possible in the same computational framework. The computation of two structures at once as a mean to exploit additional constraints is a principle that is used occasionally in computer vision, and it has been employed in the past for inferring structure and motion (e.g., [四]), motion and depth (e.g., [⿴囗木]), optical flow and camera calibration (e.g., [四]), etc. In our case, this "one shot" approach is shown to improve the result of both inference problems, and in particular, to bring us one step closer to a practical shape from specular flow algorithm even in the presence of parabolic lines and parabolic singularities and directly from image sequences.

\section{Simultaneous specular flow and surface estimation}

Consider the now classical variational approach for optic flow (e.g. [ $[\mathbf{Q}, \mathbf{Q}, \mathbf{\square}]$ ) but incorporate into the energy functional constraints between the sought-after specular flow and the unknown surface that gives rise to it. Formally, we proposed to consider an energy minimization problem of the following type 


$$
\underset{\mathbf{u}, \mathbf{r}}{\operatorname{argmin}} E(\mathbf{u}, \mathbf{r})=\underset{\mathbf{u}, \mathbf{r}}{\operatorname{argmin}} \int_{\Omega} E_{\text {flow }}(d I, \mathbf{u})+E_{\text {surface }}(\mathbf{u}, \mathbf{r}) d \mathbf{x}
$$

where $\Omega$ is the image domain, $d I$ represents image derivatives (at any desired order), and $\mathbf{u}$ and $\mathbf{r}$ are the sought-after specular flow and specular surface, respectively. In order to formulate the constraints between the unknown surface and flow, one should choose an imaging model. We follow the model proposed in the SFSF literature [ $\square, \square, \boldsymbol{\square}, \square]$, to enjoy the advantages of the linear SFSF equation (Eq. 1).

\subsection{The basic energy functional}

Let us now develop the $E_{\text {flow }}$ and $E_{\text {surface }}$ components for Eq. 2. Clearly, the specular flow, being an optical flow, should satisfy the popular brightness constancy assumption (BCA) [प]. As a regularization, let us also apply the standard piecewise smoothness constraint ${ }^{1}$. Additionally, let both these constraints considered under a robust panelizer function $\psi$ to better handle outliers and permit discontinuities in the flow [0]. We obtain:

$$
E_{B C A}=\psi\left((I(x+u, y+v, t+1)-I(x, y, t))^{2}\right) \quad E_{\text {smooth }_{f}}=\psi\left(\|\nabla u\|^{2}+\|\nabla v\|^{2}\right) .
$$

Moving on to consider the surface, the basic constraint here is derived from the linear SFSF equation [ $[\theta]$ which expresses the relation between the flow, the axis rotation $\omega=\left(\omega_{1}, \omega_{2}, \omega_{3}\right)$ and the surface. By manipulating Eq. 1 and using robust function $\psi$ we obtain

$$
E_{r e f}=\psi\left(\left(\nabla r^{a} \cdot \mathbf{u}-\omega_{3} r^{b}+\omega_{2} r^{c}\right)^{2}\right)+\psi\left(\left(\nabla r^{b} \cdot \mathbf{u}+\omega_{3} r^{a}-\omega_{1} r^{c}\right)^{2}\right)+\psi\left(\left(\nabla r^{c} \cdot \mathbf{u}-\omega_{2} r^{a}+\omega_{1} r^{b}\right)^{2}\right) .
$$

where $\mathbf{r}=\left(r^{a}, r^{b}, r^{c}\right)$ is the reflection vector field expressed by its three coordinates at each point. Next, we apply similar regularization to the surface by minimizing either its first or second derivatives. We also remember that $\mathbf{r}$ must be unit length, one can regularize by

$$
E_{\text {smooth }_{r}}=\psi\left(\|\Delta \mathbf{r}\|^{2}\right) \quad E_{\text {coherence }_{r}}=\left(\|\mathbf{r}\|^{2}-1\right)^{2} .
$$

Note that no robust function is incorporated into $E_{\text {coherencer }_{\text {r }}}$ since no slack is desired in satisfying the unit length constraint.

Finally, the surface boundaries can also be exploited since the geometry of specular reflection dictates that under orthographic projection the reflection vectors along the occluding boundary must be $(0,0,-1)$. Putting it all together we obtain the following problem

$$
\begin{aligned}
\underset{\mathbf{u}, \mathbf{r}}{\operatorname{argmin}} E(\mathbf{u}, \mathbf{r}) & =\underset{\mathbf{u}, \mathbf{r}}{\operatorname{argmin}} \int_{\Omega} E_{B C A}+\alpha_{1} E_{\text {smooth }_{f}}+\beta\left(E_{\text {ref }}+\alpha_{2} E_{\text {smooth }_{r}}\right)+E_{\text {coherence }_{r}} d \mathbf{x} \\
\text { s.t. } & \mathbf{r}(\mathbf{x})=(0,0,-1) \quad \forall \mathbf{x} \in \partial \mathbf{r} .
\end{aligned}
$$

In this equation, the parameters $\alpha_{i}$ control how deep would be the flow and surface regularizations while $\beta$ represents the confident in the reconstructed surface (which can change over the time course of the computation).

Concluding this discussion, solving Eq. 6 is the heart of our proposed approach. Although this appears to be a complicated exercise, we soon demonstrate how it can be done in ways similar to previous methods from the optical flow literature (e.g., []]), with complexity different only by a constant factor.

\subsection{Optimization and implementation}

Recall that our goal is to find $\mathbf{u}$ and $\mathbf{r}$ that minimize Eq. 6 when the input includes two consecutive image frames (induced by a known rotation vector $\omega$ ). The standard way of doing

\footnotetext{
${ }^{1}$ At this point this is a deliberate act of compromise, since it was already argued in the past that such a regularizer models parabolic singularities very poorly [0]. We return to this issue in Sec. 5.
} 
so is solving the corresponding Euler Lagrange (EL) equations that describe the necessary conditions for an extremum of the functional. For better readability, we assuming that $\psi$ in $E_{r e f}$ is quadratic, and we can write the EL equations of Eq. 6 as follows

$$
\begin{array}{r}
\psi^{\prime}\left(I_{u}^{2}\right) I_{x} I_{u}+\beta\left(\left|\mathbf{r}_{x}\right|^{2} u+\mathbf{r}_{x} \cdot \mathbf{r}_{y} v+\zeta(x)\right)-\alpha_{1} \operatorname{div}\left(\psi^{\prime}\left(|\nabla u|^{2}+|\nabla v|^{2}\right) \cdot \nabla u\right)=0 \\
\psi^{\prime}\left(I_{u}^{2}\right) I_{y} I_{u}+\beta\left(\left|\mathbf{r}_{y}\right|^{2} v+\mathbf{r}_{x} \cdot \mathbf{r}_{y} u+\zeta(y)\right)-\alpha_{1} \operatorname{div}\left(\psi^{\prime}\left(|\nabla u|^{2}+|\nabla v|^{2}\right) \cdot \nabla v\right)=0 \\
-\omega_{3} \rho_{b}+\omega_{2} \rho_{c}-\left(\rho_{a} u\right)_{x}-\left(\rho_{a} v\right)_{y}+2 r^{a}\left(|\mathbf{r}|^{2}-1\right)-\alpha_{2} \operatorname{div}\left(\psi^{\prime}\left(|\nabla r|^{2}\right) \cdot \nabla r^{a}\right)=0 \\
\omega_{3} \rho_{a}-\omega_{1} \rho_{c}-\left(\rho_{b} u\right)_{x}-\left(\rho_{b} v\right)_{y}+2 r^{b}\left(|\mathbf{r}|^{2}-1\right)-\alpha_{2} \operatorname{div}\left(\psi^{\prime}\left(|\nabla r|^{2}\right) \cdot \nabla r^{b}\right)=0 \\
-\omega_{2} \rho_{a}+\omega_{1} \rho_{b}-\left(\rho_{c} u\right)_{x}-\left(\rho_{c} v\right)_{y}+2 r^{c}\left(|\mathbf{r}|^{2}-1\right)-\alpha_{2} \operatorname{div}\left(\psi^{\prime}\left(|\nabla r|^{2}\right) \cdot \nabla r^{c}\right)=0
\end{array}
$$

where $\zeta(*)=\left(\omega_{3} r^{b}-\omega_{2} r^{c}\right) r_{*}^{a}+\left(\omega_{1} r^{c}-\omega_{3} r^{a}\right) r_{*}^{b}+\left(\omega_{2} r^{a}-\omega_{1} r^{b}\right) r_{*}^{c}, \quad * \in x, y$

$$
\begin{array}{ll}
I_{*}=\partial_{*} I(x+u, y+v, t+1), & I_{u}=I(x+u, y+v, t+1)-I(x, y, t) \\
\rho_{a}=\nabla r^{a} \cdot \mathbf{u}-\omega_{3} r^{b}+\omega_{2} r^{c}, & \rho_{b}=\nabla r^{b} \cdot \mathbf{u}+\omega_{3} r^{a}-\omega_{1} r^{c}, \quad \rho_{c}=\nabla r^{c} \cdot \mathbf{u}-\omega_{2} r^{a}+\omega_{1} r^{b}
\end{array}
$$

As can be suspected, solving these equations as is may be too complicated and numerically sensitive. We therefore propose to solve them using a dual fixed point iteration. More precisely, we suggest to iterate over two computations - one in which we fix the reflection vectors (i.e., the description of the surface) while estimating the flow, and another where we fix the flow while estimating the reflection vectors. While this stabilizes the computation, it does not deal with the non convexity of the problem and hence, similar to previous optical flow algorithms (i.e., [ $[$ ] $]$ ), we employ a multi-scale approach to avoid local minima. In each scale the EL equation is linearized, represented as a sparse matrix, and solved efficiently using iterative linear solvers. Note that in the worst case the size of this matrix can be $(3 N)^{2}$ where $N$ is the number of pixels. This size is only a constant factor larger than the $(2 N)^{2}$ size in [ $[$ ] and the numbers of non zero entries is also larger by a constant factor. Empirically, the time spent on the computation is not significantly different either, despite the apparently more complex expressions.

Like all fixed point iterations, initial guess must be provided to jump-start the computation. Fortunately, the multi-scale approach allows very rough initial guesses. In our implementation, we set the initial specular flow field $\mathbf{u}$ simply to zero. The initial guess for the reflection field $\mathbf{r}$ is a set to those vectors induced by a bounding sphere to the shape to be recovered. Indeed, different initial spheres might result in different recovered specular objects, as would be expected from the ambiguity of solutions up to a certain family of surfaces (see Sec. 6). Finally, incorporating the boundary constraints, we simply set all reflection vectors along the boundary points to $(0,0,-1)$ at each scale of the pyramid. Note that unlike previous algorithms for shape from specular flow (cf. [, $\mathbf{Q}]$ ), our suggested algorithm does not require any initial conditions for the unknown specular shape.

\section{The challenge of parabolic singularities}

Our evaluation shows that the basic algorithm suggested above successfully estimates the flow and recovers the surface's structure, as long as the latter does not contain parabolic lines (see Figs. 1 and 3). When the surface in question does contain parabolic curves, and the corresponding specular flow exhibits parabolic singularities, the suggested method perform poorly, similar to the application of any of the original SFSF algorithms [ $\square, \square, \boldsymbol{\square}, \square]$ on specular flows measured with any available optical flow algorithm. Two reasons stand out for this failure. The first is the effect of standard optical flow regularizations and the second being the destructive influence of the standard application of the multi-grid approach.

As already discussed recently in [ $[$ ] $]$, the piecewise smoothness assumption used so often in the optical flow literature conflicts the nature of the specular flow. Indeed, while the former prefers smooth behavior and slow variations almost everywhere, specular singularities exhibits dramatic growth in flow magnitude combined with a sudden 180 degree orientation 
change. As a result, most optical flow algorithms tend to underestimate the flow's magnitude in these regions, and frequently damps it to zero in order to generate a consistent solution (according to the model). This results in global and qualitative distortions in flow estimations which entail severe shape estimation in subsequent SFSF algorithms. In our case, these two interdependent effects manifest themselves at the same time in the same algorithm.

In addition to the effect of bad regularization, the implementation using multi-scale approach confounds the computation further. To understand this, consider the flow structure along a curve crossing a parabolic singularity. The flow components, $u$ and $v$, are growing fast along this curve till the curve is crossing the parabolic region. At that point, the sign of each component is flipped. In the sampled discrete flow this induces some unknown large discontinuity in each component. Consider now the interpolation of flow vectors when moving between different levels of the multi-scale pyramid. Clearly, flow samples across the singularity will cancel out in the interpolation and yield small values for the next level. Hence, at end in the proximity of the parabolic singularity, flow vectors will be rather small instead of representing the genuine structure. Needless to say that these errors propagate even more powerfully to the computation of flow derivatives around parabolic singularities, which inject additional noise and instabilities to the numerical calculations.

\section{An enhanced one-shot algorithm}

As may be observed from the description above, much of our problems with the basic algorithm relate to the magnitude and orientation aspects of the flow. This suggests that perhaps the thinking of the flow in the traditional Cartesian representation is wrong, where a shift in representation may solve some of the problems. Recently, we have advocated such a shift in the representation of motion from Cartesian to the polar coordinate system [⿴囗十. We have demonstrated its advantages especially when the estimated motion vector field is relatively complex, as indeed is the case with specular flows. Furthermore, we have argued that the polar representation preserves the independence of the magnitude and orientation components more naturally. We have also demonstrated that the statistical and physical properties of the flow field can be integrated into the energy functional more directly in the polar representation. Hence this approach allows handling motion fields of different nature much more easily and accurately. In the following we exploit these advances to propose an enhanced one-shot algorithm that estimates specular flow and specular shape simultaneously while addressing the two problems from Sec. 4, i.e., even if the shape contains parabolic lines and the flow includes parabolic singularities.

Once a specular flow is represented in polar coordinates, it is possible to see from the statistics of its two components (i.e., magnitude and orientation) that their derivatives behave differently and hence deserve different penalizer functions and regularizations [ $\theta$ ]. At the same time, the polar representation makes the use of such different functions quite straight forward. Here we take advantage of these possibilities by regularizing the flow's orientation to a typical piecewise smooth behavior and hence using its first derivative. At the same time, we would like to permit the flow's magnitude much greater variations (or else parabolic singularities are inhibited and distorted) and hence would prefer to regularize it via its second derivative. We therefore reformulate the classical Horn and Schunck [ $\square$ ] optical flow functional using polar coordinates and according to the observations just made. We obtain [ $⿴ 囗 十$ ]

$$
\begin{aligned}
& E(\theta(x, y), m(x, y))=\int_{\Omega} \psi(I(x+m \cos \theta, y+m \sin \theta, t+1)-I(x, y, t)) \\
& +\alpha_{\theta} \psi\left(\nabla\|\theta\|^{2}\right)+\alpha_{m} \psi_{m}\left(\|\Delta m\|^{2}\right) d \mathbf{x}
\end{aligned}
$$$$
\text { such that } m>0
$$

where $\theta(x, y)$ and $m(x, y)$ are the orientation and magnitude of the flow, respectively, $\psi_{\theta}$ and $\psi_{m}$ are robust penalty functions (which could be different due to the prior statistics) 
and $\alpha_{\theta}$ and $\alpha_{m}$ are weight parameters that control the desired regularization strength in each components. To simplify the optimization of Eq. 8, we farther define the following equivalence relation $\sim$ over the values of $m$ and $\theta$. Moreover, to avoid the problems due to the periodic nature of $\theta$ we further re-formulate Eq. 8 by over parameterizing the orientation component with two auxiliary projections that must remain coupled via an additional flow

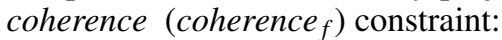

$$
\left.\begin{array}{l}
s(x, y)=\sin \theta(x, y) \\
c(x, y)=\cos \theta(x, y)
\end{array}\right\} \text { subject to } s^{2}+c^{2}=1 \quad(m, \theta) \sim \begin{cases}(m, \theta) & m \geq 0 \\
(-m, \theta+\pi) & m<0\end{cases}
$$

Now we can re-write Eq. 8 as a minimization problem:

$$
\begin{array}{r}
E_{\text {flow }_{p}}=\int_{\Omega} \psi(I(x+m \cos \theta, y+m \sin \theta, t+1)-I(x, y, t)) \\
\quad+\alpha_{\theta} \psi\left(\|\nabla \theta\|^{2}\right)+\alpha_{m} \psi_{m}\left(\|\Delta m\|^{2}\right)+\left(s^{2}+c^{2}-1\right)^{2} d \mathbf{x}
\end{array}
$$

We stress that the flow coherence constraint is a key component of the minimization problem since it ensures that variables $s$ and $c$ are true over-representation of orientation.

Changing the representation delivers one more desired benefit since now the interpolation between different levels of the multi-grid pyramid need not act on neighboring scalars of different sign, hence avoiding the destructive phenomenon observed in Sec. 4. Combining it all, we propose to to consider an energy minimization problem that expresses Eq. 6 in the polar coordinate system:

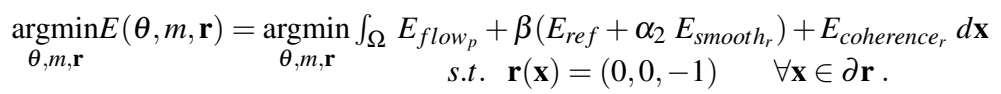

Finally, to increase the numerical stability of the proposed method, we suggest to avoid dealing with unbounded magnitude when estimating the surface. We rephrase the problem via the (always well-defined) inverse flow magnitude $\eta=1 / \mathrm{m}$. In regions with extremely small magnitude (less then 0.1 pixel) there is only little hope to estimate the flow accurately Moreover, the flow does not carry enough information for the reconstruction task. Therefore, one can set $\eta$ in these regions to zero and relay on the surface smoothness regularization that provides some compensation when recovering the surface. In practice, it is simple to make this adjustment since the flow and the surface are optimized separately. The flow estimation iteration remains as before but the reflection vector field estimation iteration (with a fixed flow) would now based on the following reformulation of $E_{\text {ref }}$

$$
\begin{aligned}
E_{r e f} & =\psi\left(\left(r_{x}^{a} c+r_{y}^{a} s-\omega_{3} \eta r^{b}+\omega_{2} \eta r^{c}\right)^{2}\right) \\
& +\psi\left(\left(r_{x}^{b} c+r_{y}^{b} s+\omega_{3} \eta r^{a}-\omega_{1} \eta r^{c}\right)^{2}\right)+\psi\left(\left(r_{x}^{c} c+r_{y}^{c} s-\omega_{2} \eta r^{a}+\omega_{1} \eta r^{b}\right)^{2}\right) .
\end{aligned}
$$
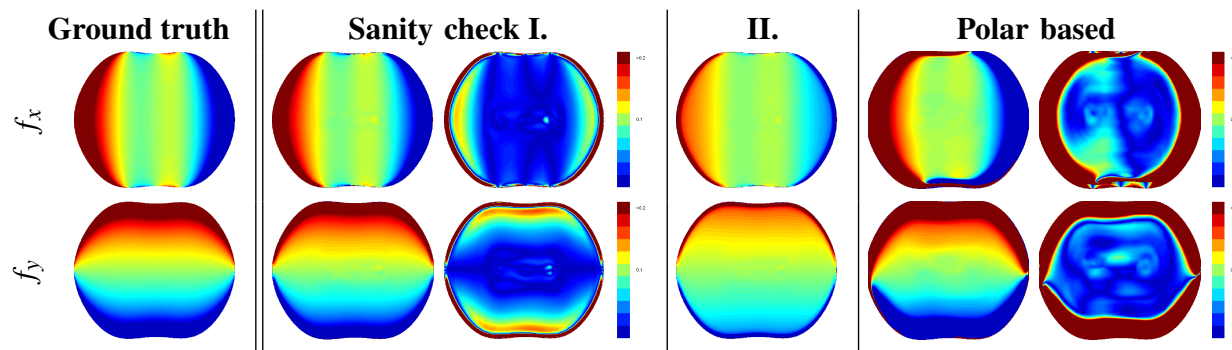

Figure 2: Sanity check I. Estimation of surface derivatives (with parabolic lines) assuming the flow is known. The average error is less than 0.1. Sanity check II. Different initial guess results in different reconstructed surface. Compare to a surface from Sanity I multiplication by 2 , the average error is less than 0.1. Polar based algorithm. Estimation of the surface derivatives using the suggested polar based algorithm. The input is only 2 images and known rotation axis. The average error is less than 0.3. See also Fig. 4. 


\section{Experiments}

With the final one-shot algorithm formulated, we validated it using real and synthetic image sequences from the only ground truth database available [ $[$ ]. In all experiments we assume that the rotation axis is known and the initial guess for the surface was a sphere. We compare the estimated flows to the known ground truth and the result obtained from a state-of-the-art optical flow algorithm $[\mathrm{⿴囗十}]$. Considering the unique properties of a specular flow, we adopted the error criteria based on the (Average) Orientation Error and the (Average) Magnitude Error [ $[$ ] $(A O E$ and $O M E$ ). To our best knowledge, no other algorithm exists to date that is able to recover a general specular surface from image sequences under the constraints of the environment being unknown and no initial conditions are provided. Hence, shape results were compared with the known ground truth data only.

To begin, a sanity check was performed by recovering the surface while the specular flow given (Fig. 2-I) and recovering the flow while the surface is given. In both cases the results are quite accurate, even in the presence of parabolic lines and singularities. We do note that the recovered surface does depart from the ground truth in certain regions where the information available from the two frames is poor; close to the surface's boundaries or where the observed motion is very small. Expectedly, surface regularization provides some compensation in these latter regions (e.g., see marked region in Fig. 3).

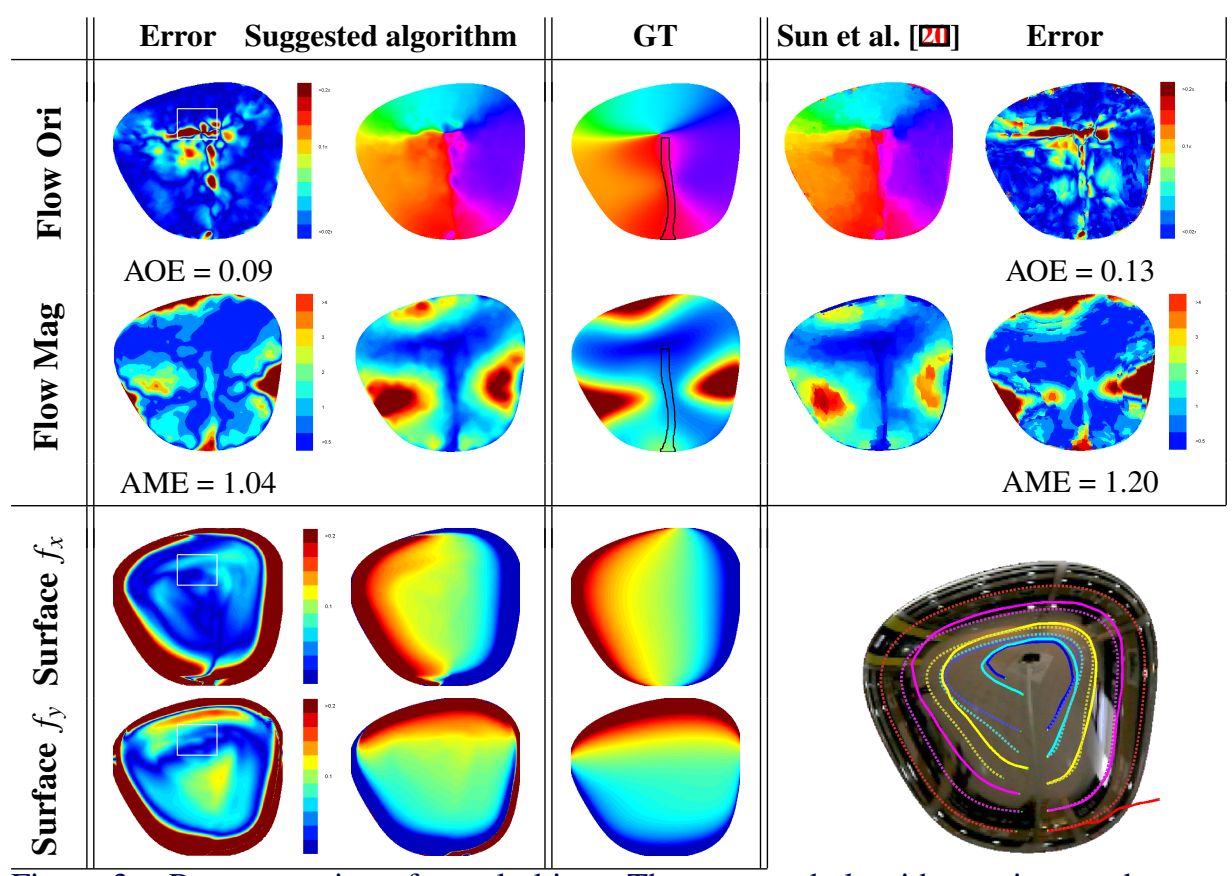

Figure 3: Reconstruction of a real object. The proposed algorithm estimates the specular flow $20 \%$ better than a state-of-the-art optical flow algorithm ([四]) and simultaneously recovers the surface quite accurately. See Fig. 1 for the reconstructed surface compared to the ground truth surface. The white box stands for regions where the orientation estimated with errors, the flow's magnitude is small and yet, the surface is estimated correctly due to the surface smoothness regularization. 
To experience the effect of the surface initial guess, Fig. 2 also repeats the same test with different initial guesses (i.e., the radius of the sphere) and indeed obtained different reconstructed surfaces. Considering the ambiguity nature of the task, this should not come as a surprise. It is straightforward to see from the generative SFSF equation [ [] that the specular flow $\mathbf{u}$ induced from a specular surface $(x, y, k f(x, y))$ under rotation axis $\omega=(0,0,1)$, is invariant to $k$, where $k$ be a positive constant. Indeed, both the first and second recovered surface derivatives have equal accuracy (below 0.1) compared to two difference surfaces where $k=1$ in the first surface and $k=2$ in the second one.

Next, we validated the complete suggested algorithm assuming nothing but the rotation axis $\omega$. We use a real image sequence from [ $[\nabla]$. However, since their acquisition device is also reflected from the object (but do not move with the environment) we treated that part of the image as outliers (marked with a black strip on the ground truth flow in Fig. 3). As can be seen, the estimation of both the flow and the surface are quite satisfactory.

To compare these results with previous methods, we selected a state-of-the-art optical flow algorithm [四], applied it on the image sequence and then ran the SFSF algorithm [ $\square]$ using standard MATLAB's ODE solvers (method ode45 and ode113) and manually provided initial shape conditions (recall that this is something our algorithm does not require). As would be expected from past observations [ $\mathrm{G}$ ], in term of flow accuracy the Sun et al. [四] algorithm estimates the flow $20 \%$ worse than our suggest method. Unfortunately, as can be seen in the lower left part of Fig. 3, this poor flow estimation was far too noisy to allow any reasonable shape reconstruction. Note the severe deviation of the estimated integral curves (solid lines) from the desired ones (dash line with the same color).

Finally, we validate the suggested algorithm using a synthetic sequence of a specular surface containing parabolic lines. As can be shown in Fig.4, the estimated flow is significantly more accurate than the state-of-the-art. The reconstruction of the surface itself is obviously more challenging than objects without parabolic lines and while our algorithm did manage to reconstruct it at least qualitatively, it did so with errors 2 times more errors compared to the object in Fig. 2. This is, however, qualitatively better the alternative (using [四], or for this matter, any optical flow algorithm followed by the SFSF approach [■]), which was not able to reconstruct any shape, despite provided initial conditions.

\section{Conclusions}

We propose a new approach that can estimate specular flow and recover the specular shape (with or without parabolic lines) that gave rise to it all in one shot. Given how all previous shape-from-specular-flow approaches require an estimation of specular flow, which present optical flow algorithm fail to reasonably prove, our suggested approach is the first one to facilitate specular shape reconstruction from real image sequences. Furthermore, contrary to all previous approaches, initial shape conditions are not needed.

Despite the above, the accuracy of the proposed approach should be improved. Natural extensions should consider better and more elaborate regularizations that address the structure of parabolic singularities more explicitly. Furthermore, it is also a practical challenge to consider the estimation of the rotation axis as part of the optimization process, perhaps at the price of increased ambiguity; the understanding of the latter should be extended beyond specific cases $[\square]$ and examples to describe the equivalence class of all surfaces and rotation axes that give rise to the same specular flow. 


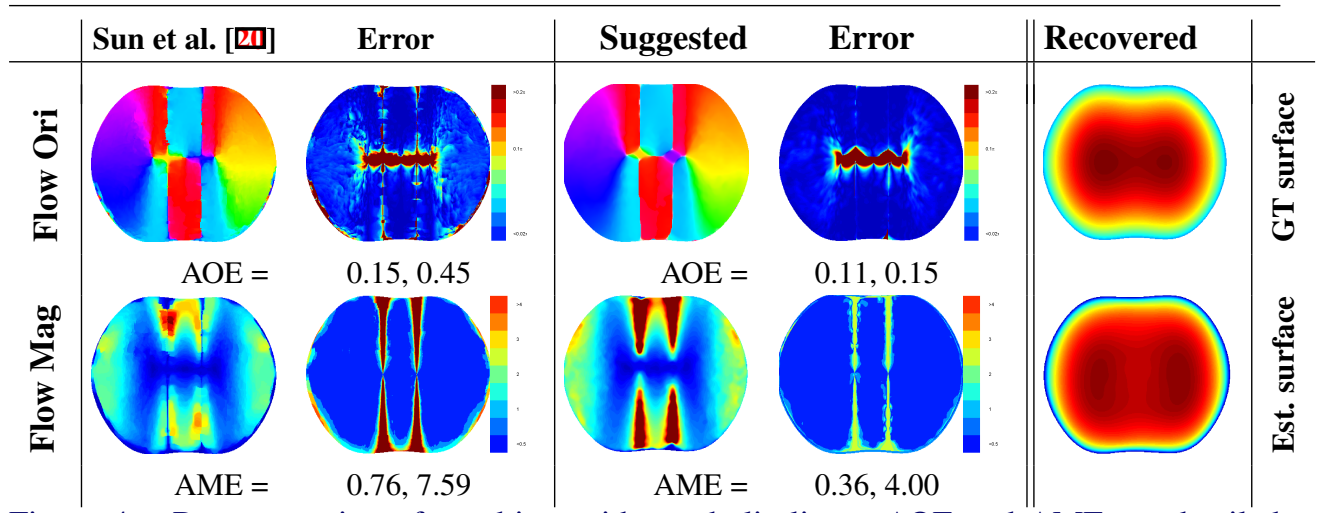

Figure 4: Reconstruction of an object with parabolic lines. AOE and AME are detailed for the entire image and for the (challenging) parabolic singularity regions. The proposed algorithm estimates the specular flow $60 \%$ better than a state-of-the-art optical flow algorithm ([四]) and simultaneously recovers the surface. See Fig. 2 for the estimated surface derivatives compared to the ground truth.

\section{Acknowledgments}

This work was funded in part by the Israel Science Foundation under grant No. 1245/08, the US Air Force European Office of Aerospace Research and Development grant number FA8655-09-1-3016, and the European Commission in the 7th Framework Programme (CROPS GA no 246252). We also thank the generous support of the Frankel Fund and the Paul Ivanier Robotics Center at Ben-Gurion University.

\section{References}

[1] Y. Adato, Y. Vasilyev, O. Ben-Shahar, and T. Zickler. Toward a theory of shape from specular flow. In Proceedings of the IEEE International Conference on Computer Vision, 2007.

[2] Y. Adato, Y. Vasilyev, O. Ben-Shahar, and T. Zickler. Shape from specular flow. IEEE Transactions on Pattern Analysis and Machine Intelligence, 32(11):2054-2070, 2010.

[3] Y. Adato, T. Zickler, and O. Ben-Shahar. Toward robust estimation of specular flow. In British Machine Vision Conference, 2010.

[4] Y. Adato, T. Zickler, and O. Ben-Shahar. A polar representation of motion and implications for optical flow. In Proceedings of the IEEE Conference on Computer Vision and Pattern Recognition, pages 1145-1152, 2011.

[5] T. Basha, Y. Moses, and N. Kiryati. Multi-view scene flow estimation: A view centered variational approach. In Proceedings of the IEEE Conference on Computer Vision and Pattern Recognition, pages 1506-1513, 2010.

[6] M.J. Black and P. Anandan. Robust dynamic motion estimation over time. In Proceedings of the IEEE Conference on Computer Vision and Pattern Recognition, pages 296-302, 1991.

[7] A. Blake and H. Bulthoff. Shape from specularities: Computation and psychophysics. Philosophical Transactions of the Royal Society B (London), 331(1260):237-252, 1991.

[8] T. Brox, A. Bruhn, N. Papenberg, and J. Weickert. High accuracy optical flow estimation based on a theory for warping. In Proceedings of the European Conference on Computer Vision, 2004. 
[9] G.D. Canas, Y. Vasilyev, Y. Adato, T. Zickler, S. Gortler, and O. Ben-Shahar. A linear formulation of shape from specular flow. In Proceedings of the IEEE International Conference on Computer Vision, 2009.

[10] R.W. Fleming, A. Torralba, and E.H. Adelson. Specular reflections and the perception of shape. Journal of Vision, 4:798-820, 2004.

[11] B.K.P. Horn and B.G. Schunck. Determining optical flow. Artificial Intelligence, 17:185-203, 1981.

[12] M.S. Longuet-Higgins. Reflection and refraction at a random moving surface. pattern and paths of specular points. Journal of the Optical Society of America, 50(9):838-844, 1960.

[13] D. Marr. Vision. W.H.Freeman and Company, 1982.

[14] M. Oren and S.K. Nayar. A theory of specular surface geometry. International Journal of Computer Vision, 24(2):105-124, 1997.

[15] S. Roth and M.J. Black. Specular flow and the recovery of surface structure. In Proceedings of the IEEE Conference on Computer Vision and Pattern Recognition, pages 1869-1876, 2006.

[16] S. Rozenfeld, I. Shimshoni, and M. Lindenbaum. Dense mirroring surface recovery from 1d homographies and sparse correspondence. In Proceedings of the IEEE Conference on Computer Vision and Pattern Recognition, 2007.

[17] A. Sankaranarayanan, A. Veeraraghavan, O. Tuzel, and A. Agrawal. Specular surface reconstruction from sparse reflection correspondences. In Proceedings of the IEEE Conference on Computer Vision and Pattern Recognition, pages 1245-1252, 2010.

[18] S. Savarese, M. Chen, and P. Perona. Local shape from mirror reflections. International Journal of Computer Vision, 64(1):31-67, 2005.

[19] J.E. Solem, H. Aanaes, and A. Heyden. A variational analysis of shape from specularities using sparse data. In Proc. 3D Data Processing, Visualization, and Transmission, pages 26-33, 2004.

[20] D.Q. Sun, S. Roth, and M.J. Black. Secrets of optical flow estimation and their principles. In Proceedings of the IEEE Conference on Computer Vision and Pattern Recognition, pages 2432 2439, 2010.

[21] L. Valgaerts, A. Bruhn, and J. Weickert. A variational model for the joint recovery of the fundamental matrix and the optical flow. In Proceedings of the Annual Symposium of the German Association for Pattern Recognition, 2008.

[22] Y. Vasilyev, Y. Adato, T. Zicker, and O. Ben-Shahar. Dense specular shape from multiple specular flows. In Proceedings of the IEEE Conference on Computer Vision and Pattern Recognition, 2008 .

[23] S. Waldon and C.R. Dyer. Dynamic shading, motion parallax and qualitative shape. In Proc. IEEE Workshop on Qualitative Vision, pages 61-70, 1993.

[24] A. Wedel, D. Cremers, T. Pock, and H. Bischof. Structure-and motion-adaptive regularization for high accuracy optic flow. In Proceedings of the IEEE International Conference on Computer Vision, pages 1663-1668, 2009.

[25] A. Zisserman, P. Giblin, and A. Blake. The information available to a moving observer from specularities. Image and Vision Computing, 7:38-42, 1989. 